\title{
Soft Tissue Infection of the Forearm With Scedosporium apiospermum Complex and Neisseria spp. Following a Dog Bite
}

\author{
Vidya S. Kollu $^{1}$, Jena Auerbach ${ }^{2}$, Alaina S. Ritter ${ }^{1}$
}

1. Department of Infectious Diseases and Global Medicine, College of Medicine, University of Florida, Gainesville, USA 2. Department of Pathology, Immunology and Laboratory Medicine, College of Medicine, University of Florida, Gainesville, USA

Corresponding author: Vidya S. Kollu, vskollu@gmail.com

\begin{abstract}
We report a case of a complex skin and soft tissue infection caused by Scedosporium apiospermum complex and Neisseria spp. following a dog bite. While Neisseria skin and soft tissue infections after dog bites have been reported, only one case of subsequent infection caused by Scedosporium spp. has been noted in the literature. To the best of our knowledge, this is the first reported case of coinfection of these particular organisms following a dog bite.
\end{abstract}

Categories: Internal Medicine, Pathology, Infectious Disease

Keywords: scedosporium apiospermum, dog bite, neisseria spp, splendore-hoeppli phenomenon

\section{Introduction}

Dog bite injuries are a common public health problem. According to a survey conducted between 2001 and 2003, the estimated incidence of dog bites in the United States is 4.5 million per year, and 885,000 require medical attention [1]. Dog bites are usually polymicrobial, and commonly isolated pathogens include Pasteurella canis, Staphylococci, Streptococci, Moraxella, Neisseria spp., Fusobacterium, Porphyromonas, Bacteroides, and Prevotella [2]. Fungal pathogens are uncommon, and there is only one reported case of Pseudallescheria boydii (now known as Scedosporium boydii) causing tenosynovitis following a dog bite [3]. Our report describes a complex skin and soft tissue infection caused by Scedosporium apiospermum complex and Neisseria spp. in the setting of a dog bite.

\section{Case Presentation}

A 58-year-old man with a past medical history of hypertension, spinal stenosis, and depression presented to the Infectious Diseases clinic with complaints of left forearm swelling and pain. He reported that five years prior to the presentation, he broke up a fight between two dogs and was bitten by both dogs on his left forearm. He returned home, washed the wounds with tap water, and used superglue to close the puncture wounds. He did not seek medical attention. Several months later, he reportedly developed a

Review began 03/20/2021 Review ended 03/26/2021 Published 03/27/2021

๑) Copyright 2021 Kollu et al. This is an open access article distributed under the terms of the Creative Commons Attribution License CC-BY 4.0., which permits unrestricted use, distribution, and reproduction in any medium, provided the original author and source are credited. pustule on the arm that ruptured and then resolved. He subsequently noted pustules periodically arising on the forearm, draining purulent material, and then scabbing. Over the next few years, he developed increasing induration and nodularity of the forearm with worsening pain and paresthesias of the fingers. He was never evaluated by a physician for these symptoms. Several months prior to his presentation, he was handcuffed, which resulted in pressure being placed on his left wrist. His left arm subsequently became very swollen and painful, which prompted him to present to the emergency department for evaluation. On evaluation, he was afebrile. His left arm was noted to be exquisitely tender with woody induration and scabbing (Figure 1). 


\section{Cureus}
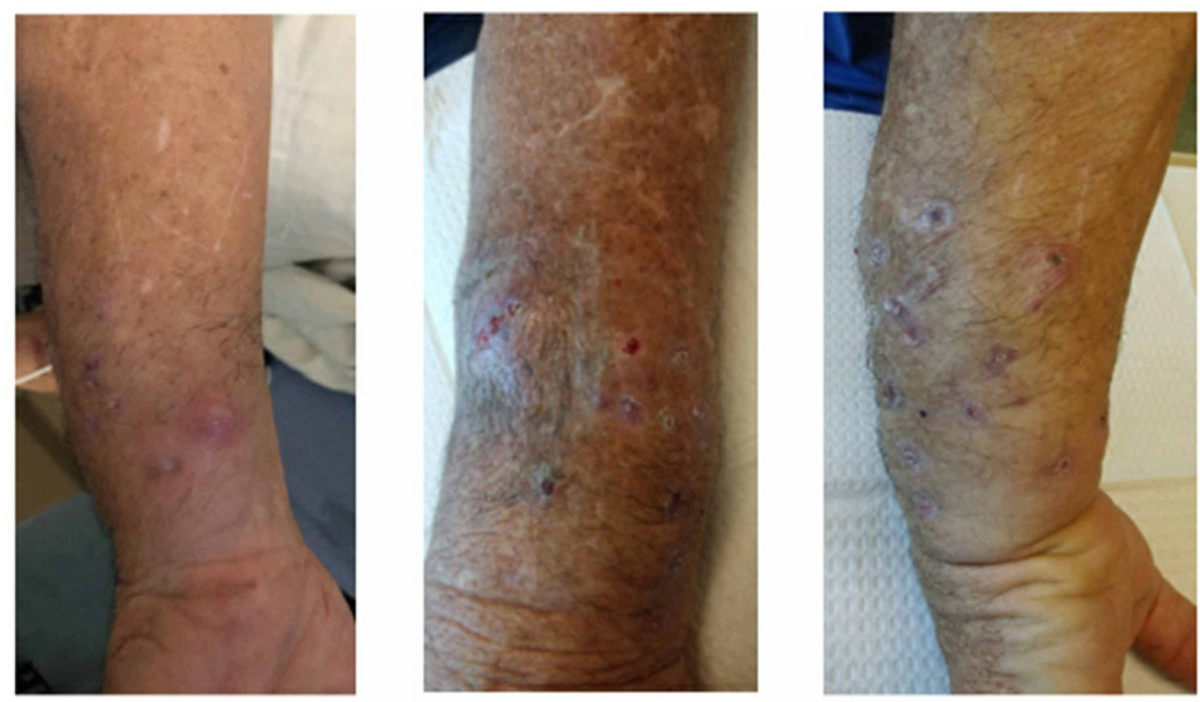

\section{FIGURE 1: Left forearm lesions prior to treatment}

Labs showed a normal white blood cell count $\left(7.3 \times 10^{3}\right.$ cells $/ \mathrm{mm}^{3}$, normal range: 4.0-10.0), normal hemoglobin (13.7 g/dL, normal range: 13.0-16.5), and normal creatinine $(0.9 \mathrm{mg} / \mathrm{dL}$, normal range: 0.38 1.02). His inflammatory markers were elevated with a high sensitivity C-reactive protein of $7.01 \mathrm{mg} / \mathrm{L}$ (normal range: $0.0-4.9$ ) and a sedimentation rate of $36 \mathrm{~mm} / \mathrm{hr}$ (normal range: $0.0-20$ ). He also had reduced sensation in the fingers of the left hand. A computerized tomography (CT) scan was obtained that showed soft-tissue edema in the hypodermis of the left forearm and the wrist compatible with cellulitis as well as focal hypoattenuation with extension into the dorsal muscle compartment that was suspicious for suppuration. Magnetic resonance imaging (MRI) done for further evaluation did not show any evidence of an abscess/drainable fluid collection or osteomyelitis, but it confirmed inflammatory changes and edema in the soft tissues (Figure 2). 


\section{Cureus}

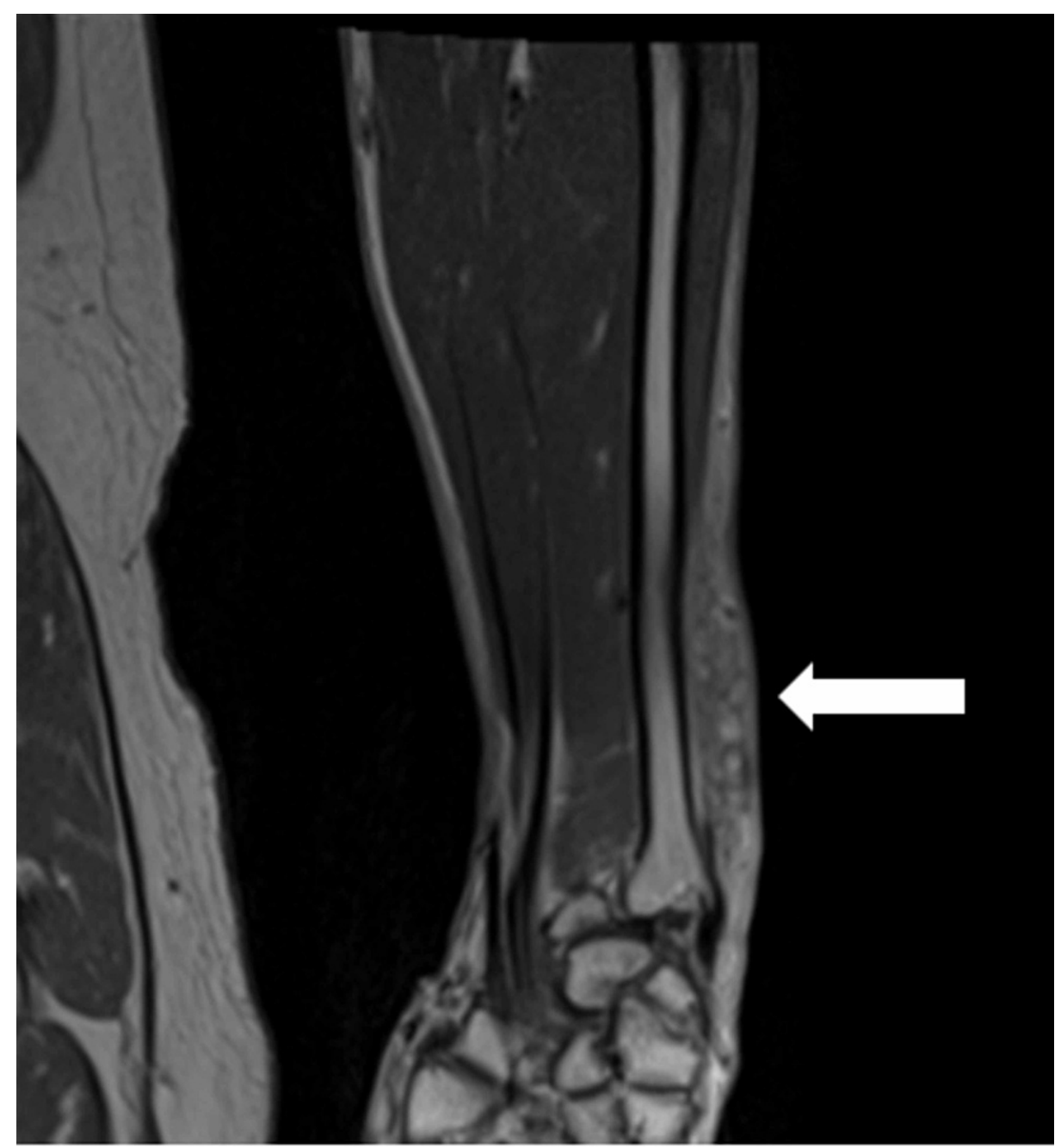

\section{FIGURE 2: MRI showing inflammatory changes and edema in the soft tissues of the left forearm}

He was discharged home on clindamycin 300 mg PO every six hours for seven days. At his orthopedic followup appointment several weeks later, he reported no improvement in the swelling or pain. He subsequently underwent an open biopsy of several lesions on the left forearm. Necrotic tissue and dense scar tissue were encountered but no purulence was noted. All the necrotic and scar tissue was excised. He was prescribed topical miconazole post-operatively. Pathology showed pseudoepitheliomatous hyperplasia, chronic inflammation, microabscesses, focal giant cells, and scar tissue. Gomori Methenamine Silver stain and Periodic Acid-Schiff stain were negative for fungal microorganisms. However, within the abscess, there was noted to be asteroid-like brightly eosinophilic material (Splendore-Hoeppli phenomenon) with focal spherical forms suggestive of fungal microorganisms (Figure 3). 


\section{Cureus}

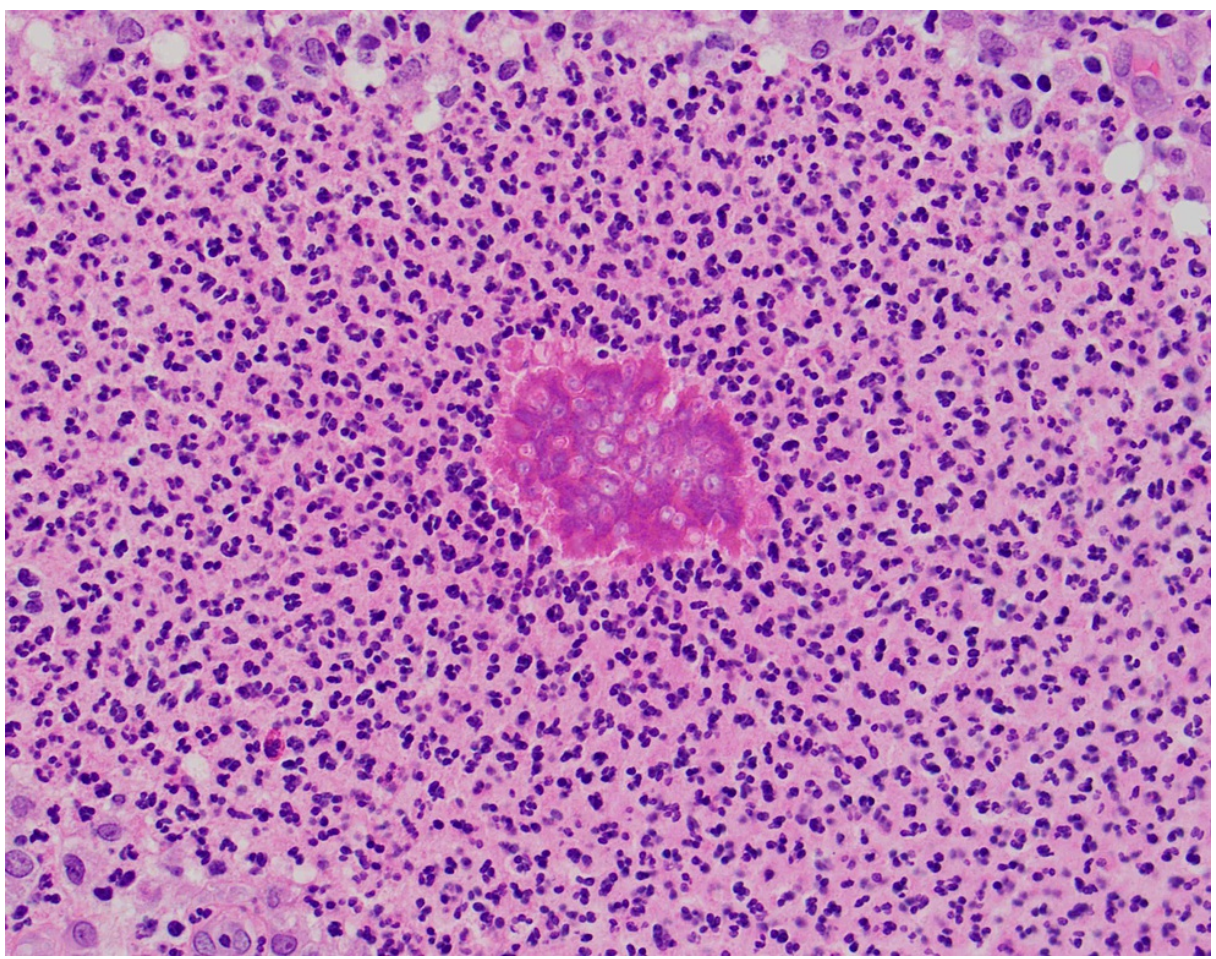

FIGURE 3: Splendore-Hoeppli phenomenon within the abscess

Cultures grew Neisseria spp. and S. apiospermum complex. Scedosporium speciation was confirmed using DNA sequencing (sensitivities in Table 1).

\begin{tabular}{|l|l|}
\hline Antibiotic & MIC \\
\hline Amphotericin B & $1 \mu \mathrm{g} / \mathrm{mL}$ \\
Anidulafungin & $4 \mu \mathrm{g} / \mathrm{mL}$ \\
Caspofungin & $1 \mu \mathrm{g} / \mathrm{mL}$ \\
Fluconazole & $16 \mu \mathrm{g} / \mathrm{mL}$ \\
Isavuconazole & $8 \mu \mathrm{g} / \mathrm{mL}$ \\
Itraconazole & $8 \mu \mathrm{g} / \mathrm{mL}$ \\
Micafungin & $0.06 \mu \mathrm{g} / \mathrm{mL}$ \\
Posaconazole & $1 \mu \mathrm{g} / \mathrm{mL}$ \\
Terbinafine & $>2 \mu \mathrm{g} / \mathrm{mL}$ \\
Voriconazole & $1 \mu \mathrm{g} / \mathrm{mL}$ \\
\hline
\end{tabular}

TABLE 1: Susceptibilities of Scedosporium apiospermum complex

Acid-fast bacteria cultures were negative at 42 days.

The patient was started on amoxicillin/clavulanate $875 \mathrm{mg} / 125 \mathrm{mg}$ by mouth twice a day to treat the Neisseria spp. and voriconazole $200 \mathrm{mg}$ by mouth twice daily to treat the S. apiospermum complex. After completing six weeks of amoxicillin/clavulanate and six months of voriconazole therapy, he experienced significant improvement in his left forearm swelling, pain, and skin lesions (Figure 4). 


\section{Cureus}
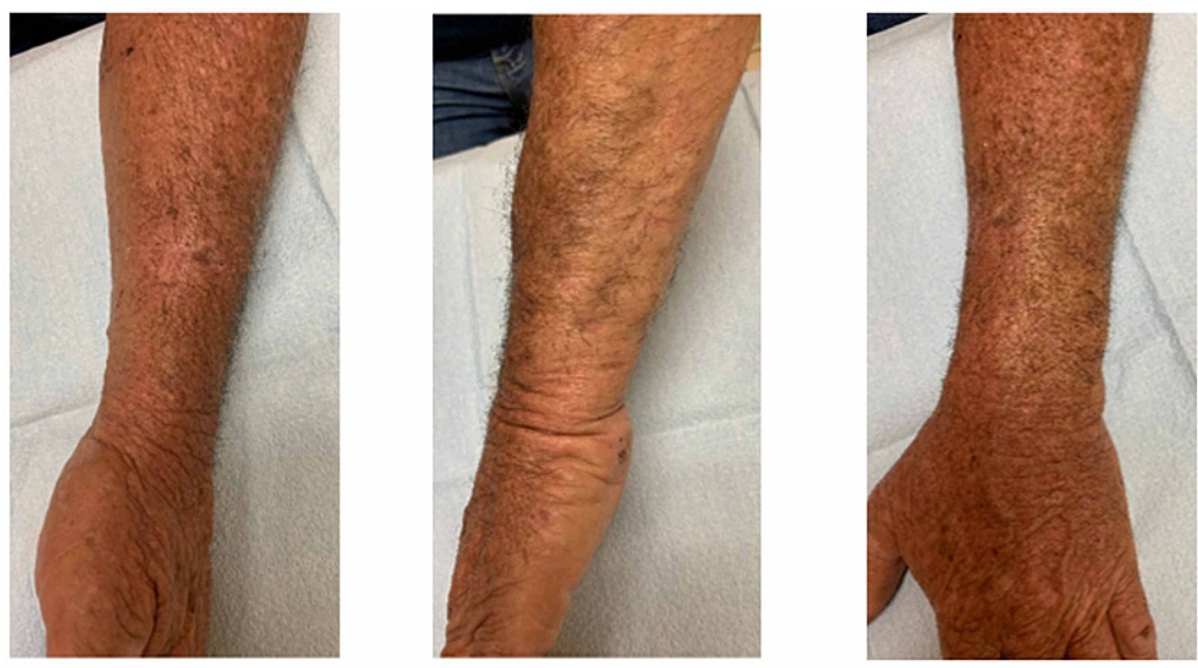

FIGURE 4: Left forearm lesions after six months of treatment

\section{Discussion}

To date, there is only one reported case of $P$. boydii (now known as S. boydii) causing tenosynovitis following a dog bite and there is no prior reported case of coinfection with Neisseria spp. and S. apiospermum complex [3]. S. apiospermum complex includes S. apiospermum, S. boydii (previously called P. boydii), $S$. angustum, S. ellipsoideum, and S. fusarium [4]. These fungi are present in freshwater, especially water that is stagnant and polluted, as well as in soil [5]. Scedosporium spp. are known to cause a wide variety of clinical infections, including mycetomas and infections of the lungs, sinuses, eye, brain, bone, joints, and heart valves [6]. These infections are usually acquired through inhalation or traumatic inoculation through the skin/epithelium [5]. Usually, the disease is localized in immunocompetent individuals but can become invasive/disseminated in immunocompromised individuals [7]. The mortality rate is often high in immunocompromised individuals, especially in the setting of pulmonary (up to $57.2 \%$ ) and central nervous system (up to $76 \%$ ) involvement [5,7]. In immunocompetent individuals, such as our patient, it can sometimes take months to years before symptoms manifest [8].

Scedosporium infection can be diagnosed by culture, direct microscopy with histochemical staining, histopathology, and DNA sequencing. It is typically diagnosed based on culture from the affected site and identified by DNA sequencing [6]. Scedosporium spp. may be resistant to amphotericin B and can break through therapy. In vitro susceptibilities to azoles are variable, and there can be reduced susceptibility to echinocandins $[9,10]$. Voriconazole is considered the drug of choice as it typically has the best in vitro activity and clinical outcomes. In isolates where voriconazole has less in vitro activity, there have been reports of successful treatment with voriconazole in combination with terbinafine or an echinocandin [11]. In a study of 107 patients treated with voriconazole, the majority of those who were successfully treated received between 13 and 799 days of treatment with a median treatment duration of 180 days. A high rate of success was noted in patients with no immunocompromising conditions [11]. Our patient did not have an underlying immunocompromising condition and improved significantly on monotherapy with voriconazole. Terbinafine was not added as there was relative resistance noted to terbinafine on sensitivity analysis. He will continue voriconazole therapy past six months, with the ultimate duration depending on clinical response.

Neisseria spp. have been described as pathogens from dog bites as early as 1974. The group includes $N$. weaveri, $N$. animaloris, and $N$. Zoodegmatis $[12,13]$. These species are considered to be normal oral flora in dogs, cats, and rodents. In humans, they primarily cause purulent wound infections but are also reported to cause pulmonary infections, chronic otitis media, endophthalmitis, tenosynovitis, and bacteremia $[12,13]$. The specific strain of Neisseria was not identified in our patient. Most strains are susceptible to amoxicillinclavulanate [14]. Our patient was treated with six weeks of amoxicillin-clavulanate, given his complex skin and soft tissue infection, and experienced appropriate clinical improvement.

\section{Conclusions}

We report a unique case of a S. apiospermum complex and Neisseria spp. coinfection after a dog bite. After six months of antifungal/antibiotic treatment, the patient experienced significant improvement of his left forearm complex skin and soft tissue infection. Thus, it is reasonable for physicians to consider these organisms as possible pathogens in patients with infections following a dog bite. Since Scedosporium spp. may have variable susceptibilities to amphotericin B, echinocandins, and azoles, sensitivity data can help guide therapy. Voriconazole is typically the drug of choice, however, given its in vitro activity. 


\section{Additional Information}

Disclosures

Human subjects: Consent was obtained or waived by all participants in this study. Conflicts of interest: In compliance with the ICMJE uniform disclosure form, all authors declare the following: Payment/services info: All authors have declared that no financial support was received from any organization for the submitted work. Financial relationships: Alaina S. Ritter declare(s) a grant from NATL INST of HLTH NIAAA. Project number 1UH2AA026214-01. Alaina S. Ritter declare(s) personal fees from DynaMed Plus. Topic editor. Other relationships: All authors have declared that there are no other relationships or activities that could appear to have influenced the submitted work.

\section{References}

1. Gilchrist J, Sacks JJ, White D, Kresnow MJ: Dog bites: still a problem? . Inj Prev. 2008, 14:296-301. 10.1136/ip.2007.016220

2. Talan DA, Citron DM, Abrahamian FM, Moran GJ, Goldstein EJ: Bacteriologic analysis of infected dog and cat bites. Emergency Medicine Animal Bite Infection Study Group. N Engl J Med. 1999, 340:85-92. 10.1056/NEJM199901143400202

3. Ruinemans GM, Haagsma CJ, Hendrix R: Tenosynovitis caused by a pseudallescheria boydii infection and symptoms of reflex sympathetic dystrophy after a dog bite. J Clin Rheumatol. 2011, 17:363-4. 10.1097/RHU.0b013e31823279dd

4. Ramirez-Garcia A, Pellon A, Rementeria A, et al.: Scedosporium and Lomentospora: an updated overview of underrated opportunists. Med Mycol. 2018, 56:102-25. 10.1093/mmy/myx113

5. Kantarcioglu AS, Guarro J, de Hoog GS: Central nervous system infections by members of the Pseudallescheria boydii species complex in healthy and immunocompromised hosts: epidemiology, clinical characteristics and outcome. Mycoses. 2008, 51:275-90. 10.1111/j.1439-0507.2008.01489.x

6. Cortez KJ, Roilides E, Quiroz-Telles F, et al.: Infections caused by Scedosporium spp. Clin Microbiol Rev. 2008, 21:157-97. 10.1128/CMR.00039-07

7. Kantarcioglu AS, de Hoog GS, Guarro J: Clinical characteristics and epidemiology of pulmonary pseudallescheriasis. Rev Iberoam Micol. 2012, 29:1-13. 10.1016/j.riam.2011.04.002

8. Lavy D, Morin O, Venet G, Maugars Y, Prost A, Berthelot JM: Pseudallescheria boydii knee arthritis in a young immunocompetent adult two years after a compound patellar fracture. Joint Bone Spine. 2001, 68:517-20. 10.1016/s1297-319x(01)00318-9

9. Carrillo AJ, Guarro J: In vitro activities of four novel triazoles against Scedosporium spp . Antimicrob Agents Chemother. 2001, 45:2151-3. 10.1128/AAC.45.7.2151-2153.2001

10. Johnson ME, Katiyar SK, Edlind TD: New Fks hot spot for acquired echinocandin resistance in Saccharomyces cerevisiae and its contribution to intrinsic resistance of Scedosporium species. Antimicrob Agents Chemother. 2011, 55:3774-81. 10.1128/AAC.01811-10

11. Troke P, Aguirrebengoa K, Arteaga C, et al.: Treatment of scedosporiosis with voriconazole: clinical experience with 107 patients. Antimicrob Agents Chemother. 2008, 52:1743-50. 10.1128/AAC.01388-07

12. Andersen BM, Steigerwalt AG, O'Connor SP, Hollis DG, Weyant RS, Weaver RE, Brenner DJ: Neisseria weaveri sp. nov., formerly CDC group M-5, a gram-negative bacterium associated with dog bite wounds. J Clin Microbiol. 1993, 31:2456-66. 10.1128/JCM.31.9.2456-2466.1993

13. Heydecke A, Andersson B, Holmdahl T, Melhus A: Human wound infections caused by Neisseria animaloris and Neisseria zoodegmatis, former CDC Group EF-4a and EF-4b. Infect Ecol Epidemiol. 2013, 3:20312. 10.3402/iee.v3i0.20312

14. Goldstein EJC, Citron DM, Tyrrell KL, Leoncio ES: In vitro activity of pexiganan and 10 comparator antimicrobials against 234 isolates, including 93 Pasteurella Species and 50 anaerobic bacterial isolates recovered from animal bite wounds. Antimicrob Agents Chemother. 2017, 61:e00246-17. 10.1128/AAC.00246-17 